\title{
AMENDMENTS
}

\section{Author Correction: A systems biology pipeline identifies regulatory networks for stem cell engineering}

Melissa A. Kinney, Linda T. Vo, Jenna M. Frame, Jessica Barragan, Ashlee J. Conway, Shuai Li, Kwok-Kin Wong, James J. Collins (1), Patrick Cahan (D), Trista E. North, Douglas A. Lauffenburger (D) and George Q. Daley (1)

Correction to: Nature Biotechnology https://doi.org/10.1038/s41587-019-0159-2, published online 1 July 2019.

In the version of this article initially published, the second NIH grant "R24-DK49216" to author George Q. Daley contained an error. The grant number should have read U54DK110805. The error has been corrected in the HTML and PDF versions of the article.

\section{Publisher Correction: Reply to 'A lack of evidence for six times more anxiety and depression in US graduate students than in the general population'}

Teresa M. Evans, Lindsay Bira and Nathan L. Vanderford (D)

Correction to: Nature Biotechnology https://doi.org/10.1038/s41587-019-0181-4, published online 24 June 2019.

In the version of this article initially published, the Editor's Note indicating that the article has been peer-reviewed was missing. The error has been corrected in the HTML and PDF versions of the article.

Published online: 9 July 2019

https://doi.org/10.1038/s41587-019-0211-2

\section{Publisher Correction: Drug pipeline 1Q19}

\section{Laura DeFrancesco}

Correction to: Nature Biotechnology https://doi.org/10.1038/s41587-019-0146-7, published online 4 June 2019.

In the version of this article initially published, in reference to Translate Bio's MRT5201, the sentence "FDA put a clinical hold on this synthetic mRNA drug encoding ornithine transcarbamylase, owing to toxicity and clearance issues" should have read "FDA put a clinical hold on this synthetic mRNA drug encoding ornithine transcarbamylase, pending more toxicology data on clearance times." The error has been corrected in the HTML and PDF versions of the article. 